\title{
Numerical Analysis for Effect of Applied Shear Stress on Longitudinal Compressive Strength of Fiber Reinforced Composite Materials
}

\author{
Takeaki Nadabe \\ Department of Advanced Energy \\ The University of Tokyo \\ Chiba, Japan \\ e-mail: nadabe@smart.k.u-tokyo.ac.jp
}

\author{
Nobuo Takeda \\ Department of Advanced Energy \\ The University of Tokyo \\ Chiba, Japan \\ e-mail: takeda@smart.k.u-tokyo.ac.jp
}

\begin{abstract}
This study investigates numerical analysis for effect of applied shear stress on longitudinal compressive strength of fiber reinforced composite materials. For this purpose, firstly numerical simulation of longitudinal compressive failure of composite materials is conducted. Simulated results show that when compressive failure initiates in the material, matrix causes large shear deformation due to nonlinear stress-strain relation of matrix, and the initiation of compressive failure of the material is closely related with the nonlinear stress-strain relation of matrix. When the shear stress is applied to the material, yield behavior of matrix is affected by the applied shear stress, and the initiation of failure of the material also changes, consequently the compressive strength of the material changes. The numerical results show that when the applied shear stress increases, the compressive strength of composite materials decreases, and this relation is close to the linear relation. The numerical results are also compared with the experimental results, and correspondence is found in between numerical and experimental results. Then the relationship between applied shear stress and compressive strength is analyzed from the mathematical equation expressing initiation of compressive failure of the material. As the results of the analysis, when the applied shear stress increases, yielding of matrix occurs at early stage, and then nonlinearity appears in the stress-strain relation of composite material, and the compressive strength of the material reduces. The analysis results of the relationship between applied shear stress and compressive strength agree with the above numerical results and experimental results.
\end{abstract}

Keywords-numerical analysis; fracture mechanism; strength analysis; composite materials

\section{INTRODUCTION}

Composite materials commonly have complex internal structures including fibers, matrix, interfaces and interlaminar regions, and when precise evaluation of fracture strength of the material is conducted, the internal fracture process in the materials is necessary to be taken into account in the numerical analysis [1-6]. In recent years, composite materials are being increasingly used in several industrial fields, and the precise evaluation of mechanical response of the material under various loading condition and environmental condition increases the necessity in design and improvement of industrial products. It is well known that the applied shear stress significantly reduces the longitudinal compressive strength of composite materials [2-5]. When composite materials are used in structural elements, often the shear stress appears in the materials, and the compressive failure of the materials occurs under the applied shear stress. Therefore to evaluate the fracture strength of structural elements, it is necessary to understand the influence of shear stress for failure and strength of the materials. This study investigates numerical analysis for effect of applied shear stress on longitudinal compressive strength of fiber reinforced composite materials. Firstly numerical simulation of compressive failure of composite materials is conducted, and then the relationship between shear stress and compressive strength is analyzed. The purpose of this study is to establish the numerical analysis method to analyze the influence of shear stress for failure and strength of the materials.

\section{NUMERICAL MODEL}

The numerical model is constructed for longitudinal compressive failure of composite material. Fig. 1 illustrates the analysis model. The white and gray elements in Fig. 1 represent fibers and matrix, respectively. The thickness of the ply in y-direction is $300 \mu \mathrm{m}$. The length in $\mathrm{x}$-direction is $500 \mu \mathrm{m}$, and the thickness in $\mathrm{z}$-direction is $100 \mathrm{~mm}$. The diameter of each fiber is set to $7.0 \mu \mathrm{m}$, and the interval of fibers is $10.0 \mu \mathrm{m}$. The fiber volume fraction of the materials is set to $60.0 \%$. The fibers are modeled as beam elements. The beam elements of fibers are Timoshenko-type beam, and have three nodes and four integration points. The cross-section of beam is modeled as circle. The one fiber placed at the center has the initial misalignment as shown in Fig. 1. The initial misalignment of the fiber is introduced using the sine function. The $\mathrm{x}$ coordinate of each node is placed regularly, and the $y$ coordinate of each node is calculated using the sine function. The other fibers are modeled as the straight lines and the fiber axial direction is parallel to the $\mathrm{x}$-direction. For simulating the bending fracture of each fiber, cohesive elements are inserted in the connection of fiber beam elements. When the high bending deformation occurs in fiber beam elements, the cohesive elements open and the fiber bending breaking is simulated in the analysis. In addition, matrix is modeled by two-dimensional plate elements. The plate elements of matrix have eight nodes 


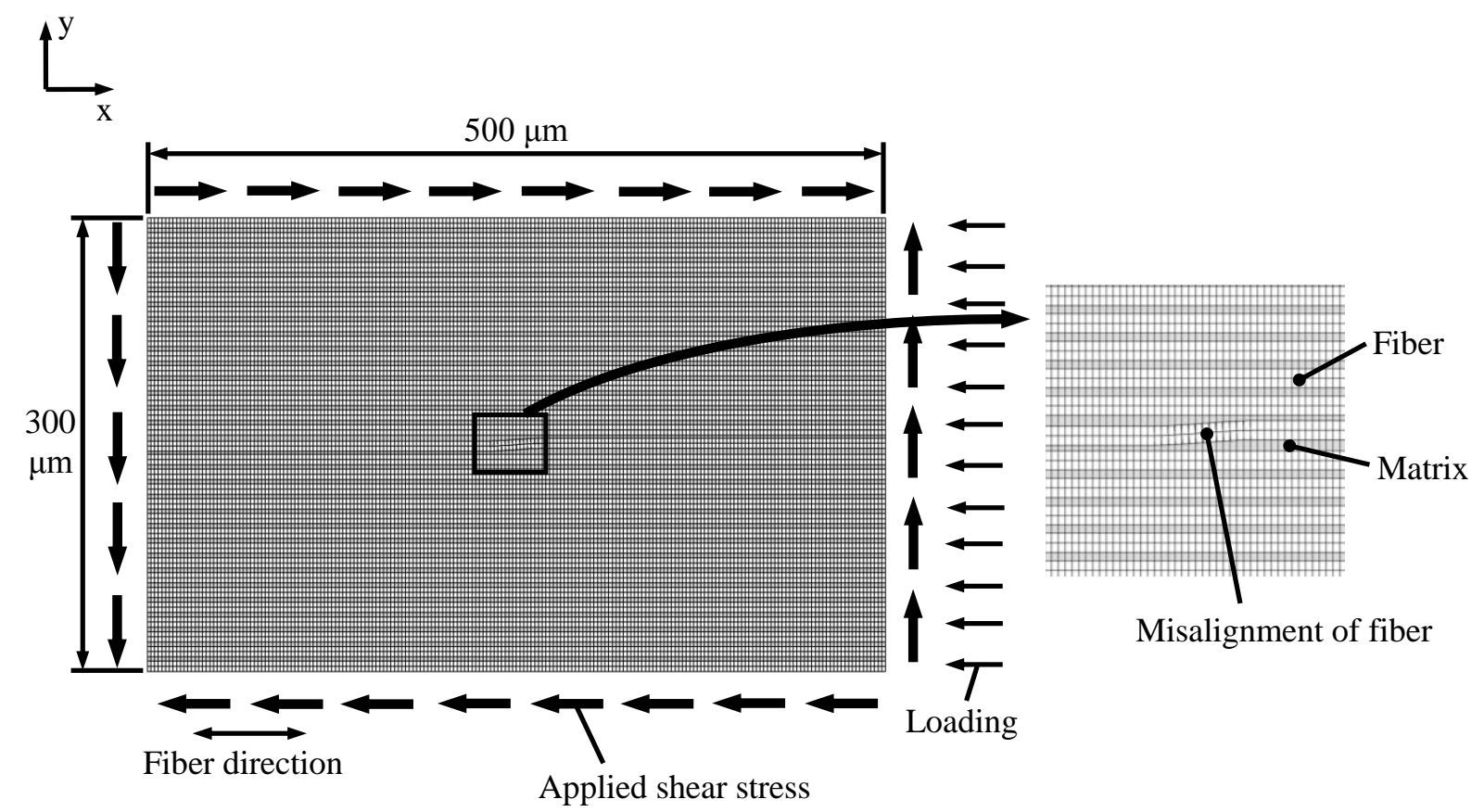

Figure 1. Numerical model for longitudinal compressive failure.

TABLE I. MAterial Property of Fiber. CARbon Fiber AS4 (HEXCEL CORP.) IS ASSUMED [7].

\begin{tabular}{|l|c|c}
\hline $\begin{array}{l}\text { Elastic modulus in fiber axial } \\
\text { direction }\end{array}$ & 225 & $\mathrm{GPa}$ \\
\hline $\begin{array}{l}\text { Elastic modulus in transverse } \\
\text { direction }\end{array}$ & 15 & $\mathrm{GPa}$ \\
\hline In-plane Poisson's ratio & 0.20 & \\
\hline In-plane shear modulus & 15 & $\mathrm{GPa}$ \\
\hline Transverse shear modulus & 7.0 & $\mathrm{GPa}$ \\
\hline
\end{tabular}

TABLE II. MATERIAL PROPERTY OF MATRIX. EPOXY RESIN 35016 (HERCULES CHEMICAL COMPANY, INC.) Is ASSUMED [7].

\begin{tabular}{|l|c|c|}
\hline Elastic modulus & 4.2 & $\mathrm{GPa}$ \\
\hline Poisson's ratio & 0.34 & \\
\hline Yield stress & 90 & $\mathrm{MPa}$ \\
\hline
\end{tabular}

and four integration points in order to avoid the shear locking and zero-energy mode deformation particularly in plastic deformation. The applied shear stress is introduced as the average stress, and the amount of the applied shear stress is changed.

Due to the atomic structure in the inside of the fibers, the fibers commonly have the different material property in between fiber axial and transverse directions. Here, the fibers are modeled by the transversely isotropic elastic material. Table I shows the material property of the fibers. Carbon fiber AS4 (Hexcel Corp.) is assumed [7]. Matrix is modeled by isotropic elastic-plastic material. Commonly the compressive failure of composite materials is affected by the nonlinear stress-strain relation of matrix, thus in this analysis the nonlinear stress-strain curve of matrix shown in Fig. 2 is applied, and the nonlinear finite element analysis is conducted. Table II shows the material property of matrix. Epoxy resin 3501-6 (Hercules Chemical

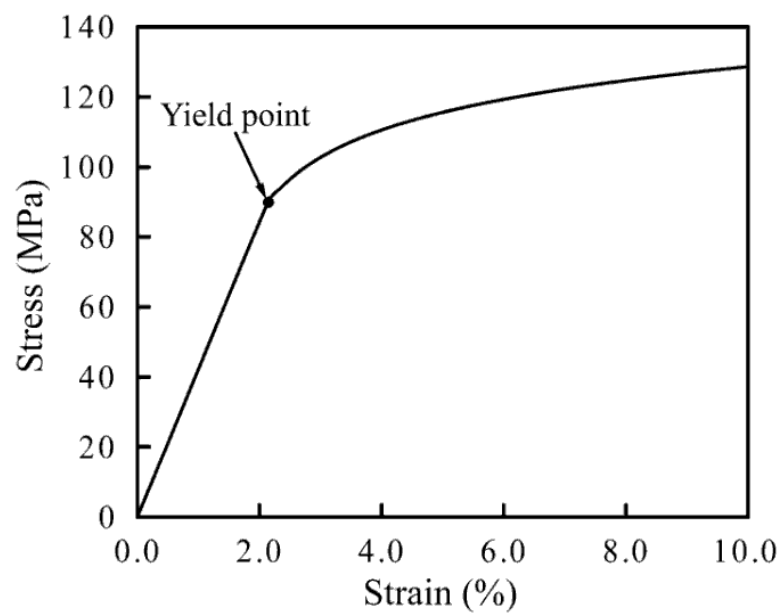

Figure 2. Stress-strain curve of matrix.

Company, Inc.) is assumed [7]. The quasi-static and room temperature environment are assumed in the analysis.

Since the geometrical nonlinearity commonly affects the buckling phenomena of the materials, the geometrical nonlinear effect is incorporated in the analysis. The incremental analysis in the finite element analysis is conducted by the arc-length method. In the initial increment, the average applied strain to the material in $\mathrm{x}$ direction is set to $0.002 \%$. The analysis is conducted until the average applied strain $8.0 \%$. The domain decomposition method is applied to conduct the parallel computing in the numerical calculation. The authors produced fortran program for this analysis, and the analysis is conducted using this program.

\section{SIMULATED RESUlTS}

Fig. 3 shows the simulated results of deformation and stress distribution in the material. At average applied strain 
Deformation

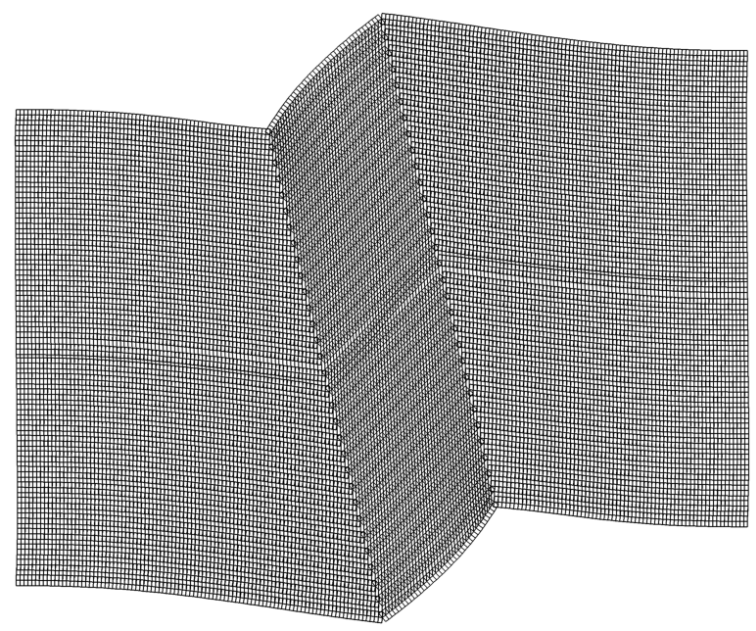

At strain $5.5 \%$

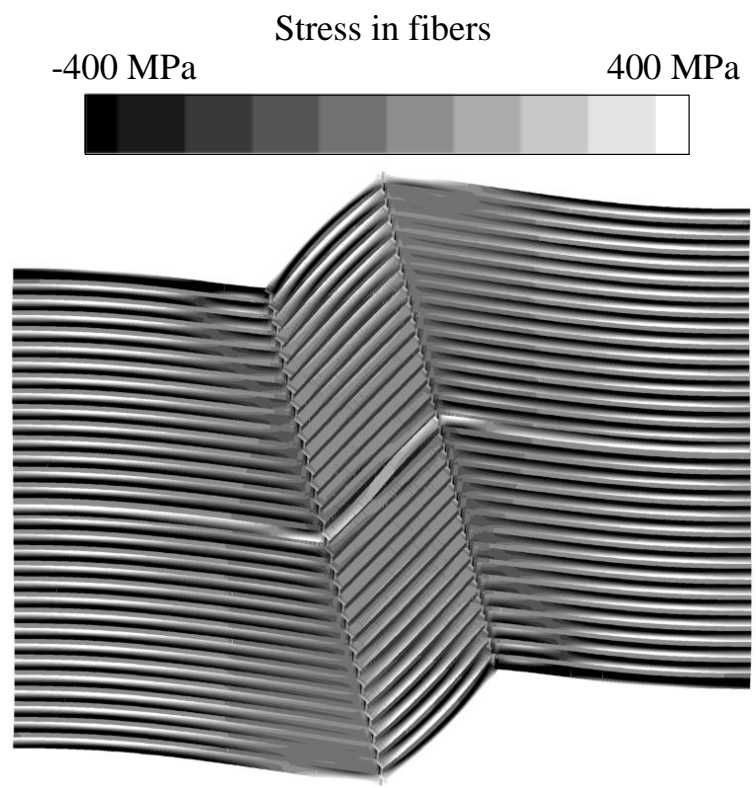

At strain $5.5 \%$
Experiment (Microscope photograph)
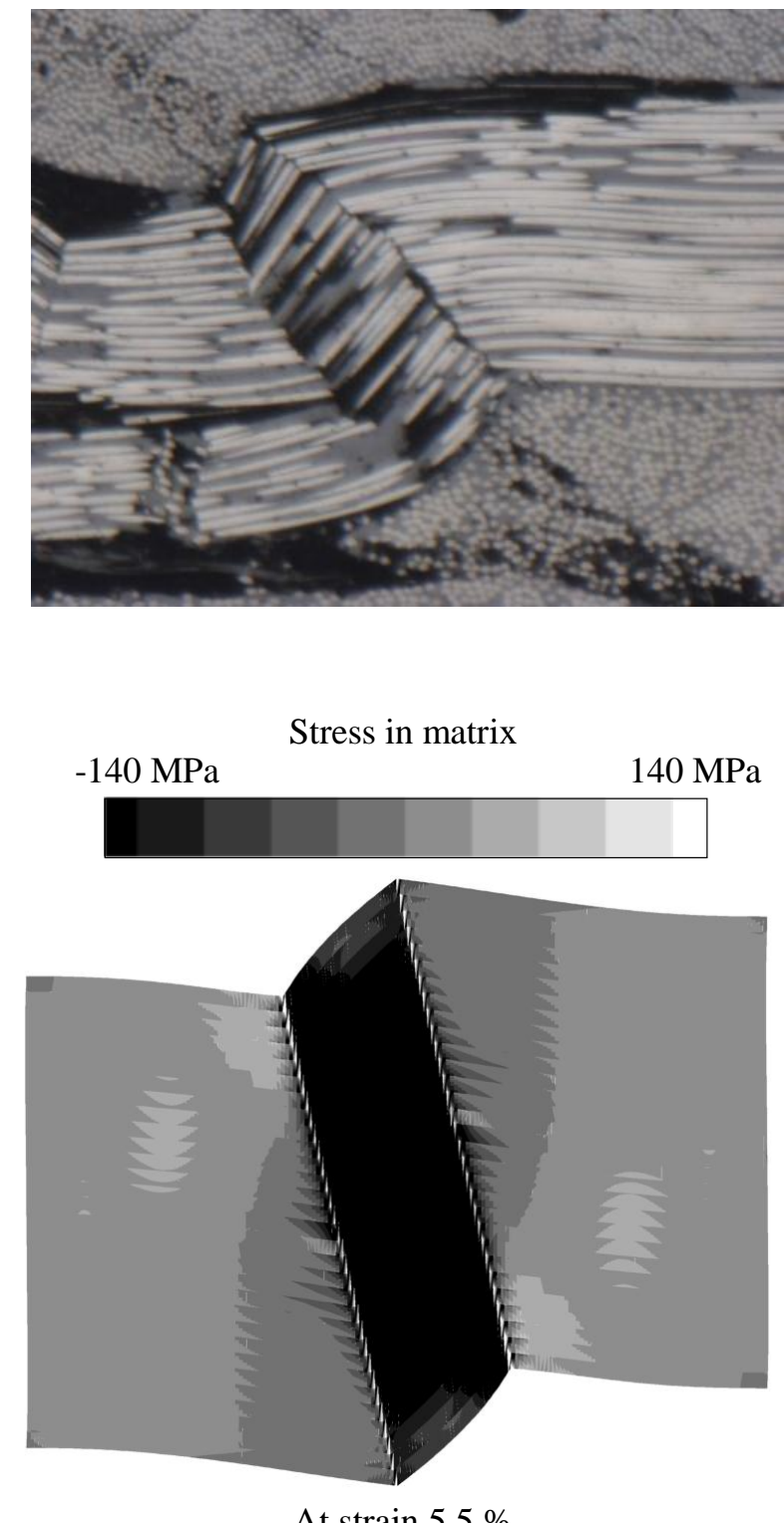

Figure 3. Simulated results of deformation and stress distribution.

$1.20 \%$, the fiber breaking occurs, and a band of broken fibers is formed, and after this moment the inclination of fibers increases with the increase of applied strain. As shown in Fig. 3, the deformation of the material after the fracture is formed is close to the microscope photograph observed in the experiment, therefore the current simulation is considered to correspond with the actual material deformation. In addition, as indicated in Fig. 3, in a band of broken fibers the fiber direction is largely rotated. Matrix in between fibers causes large shear deformation. After the yielding of matrix, the nonlinear shear stiffness of matrix due to nonlinear stress-strain relation of matrix significantly reduces because of the yielding, and the shear strain rapidly increases. Then the shear deformation of this part of matrix increases, and due to the shear deformation of the part, the failure is formed in the material. The reduction of nonlinear shear stiffness of matrix after yielding due to nonlinear stress-strain relation of matrix is the essential factor in the onset of the failure of the material as it causes large increase of shear deformation of matrix.

When the shear stress is applied to the material, yield behavior of matrix is affected by the applied shear stress, and the initiation of failure of the material also changes, consequently the material strength in compressive failure changes.

In order to compare the simulated results for relationship between applied shear stress and compressive strength of the material with the experimental results, two kinds of composite material carbon fiber/epoxy resin T300/BSL914C and carbon fiber/vinylester resin IM-712K/Derakane 411-C50 are analyzed. The simulated results for this relationship are shown in Fig. 4(a) and (b). The experimental results in Fig. 4(a), (b) are shown in Ref. [8-9]. The simulated result of uniaxial compressive strength changes depending on the angle of initial misalignment of fiber which is determined when the analysis model is constructed before analysis starts, and 


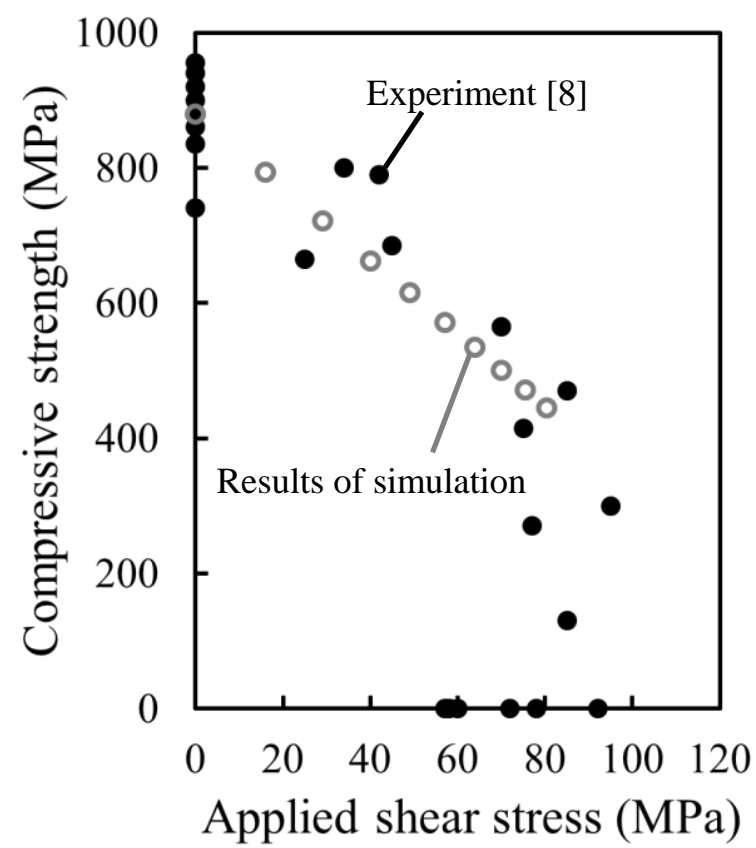

(a) Carbon fiber/epoxy resin T300/BSL914C

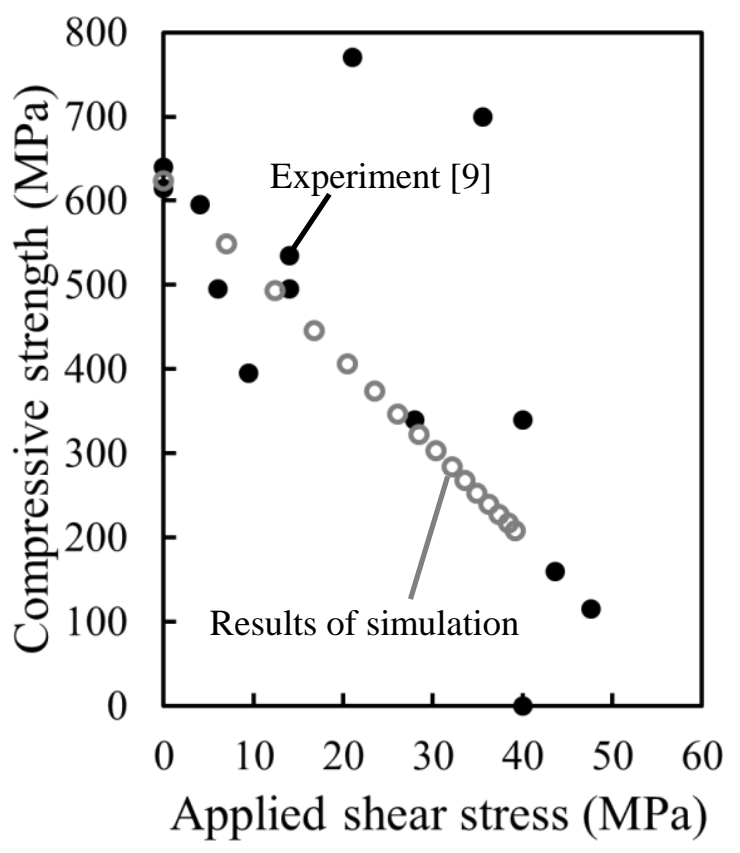

(b) Carbon fiber/vinylester resin IM-7-12K/Derakane 411-C50

Figure 4. Simulated results for relationship between applied shear stress and compressive strength of the material.

because of this the angle of initial misalignment is determined so that the analysis result of uniaxial compressive strength fits with the actual value of material strength. Therefore, firstly the uniaxial compressive strength is set to the actual material strength value, and the associated initial misalignment angle value is also set, then using this analysis model, the influence of applied shear stress is investigated in the analysis. As the results of analysis, when the applied shear stress increases, the compressive strength of composite materials decreases, and this relation is close to the linear relation as shown in Fig. 4(a) and (b). This relation including the reduction of strength and the linear relation is also observed in experimental results as shown in Fig. 4(a) and (b).

\section{ANALYSIS USING MATHEMATICAL EQUATION} EXPRESSING DEFORMATION OF COMPOSITE MATERIALS

The deformation of composite materials is expressed by the following two equations which are motion equation and constitutive equation [10]

$$
\begin{gathered}
\rho_{0} \frac{\partial^{2} u_{i}}{\partial t^{2}}=\frac{\partial P_{i j}}{\partial X_{j}}+\rho_{0} f_{i} \\
\dot{P}_{i j}=J \frac{\partial X_{j}}{\partial x_{m}}\left(C_{i m k l}^{m a t}+\sigma_{l m} \delta_{i k}\right) \frac{\partial \dot{u}_{k}}{\partial x_{l}}
\end{gathered}
$$

where $\rho_{0}$ is density, $t$ is time, $u_{i}$ is displacement, $X_{j}$ is coordinate at reference configuration, $P_{i j}$ is the first PiolaKirchhoff stress, $f_{i}$ is external force, $F_{i j}$ is deformation gradient, $J=\operatorname{det} F_{i j}$ is Jacobian, $x_{i}$ is coordinate at present configuration, $C_{i m k l}^{m a t}$ is constitutive tensor in material description, $\sigma_{l m}$ is Cauchy stress and $\delta_{i k}$ is Kronecker delta. Equations (1) and (2) are unified to one equation as the following,

$$
\rho_{0} \frac{\partial^{2} \dot{u}_{i}}{\partial t^{2}}-\rho_{0} \dot{f}_{i}=\frac{\partial}{\partial X_{j}}\left(A_{i j k l} \frac{\partial \dot{u}_{k}}{\partial x_{l}}\right)
$$

where tensor $A_{i j k l}$ is represented as the following

$$
A_{i j k l}=J \frac{\partial X_{j}}{\partial x_{m}}\left(C_{i m k l}^{m a t}+\sigma_{l m} \delta_{i k}\right)
$$

In (4), when the following condition is satisfied, failure appears in the material [10].

$$
\operatorname{det}\left(A_{i j k} n_{j} n_{l}\right)=0
$$

where $n_{j}$ is a vector which is related with the resultant material deformation after failure. Here the numerical analysis is conducted for the actual material property using (5). In order to obtain the material strength value from the analysis using (5), the incremental analysis is conducted. As the initial condition, stress is set to zero. Then the stress is incrementally applied. In each increment, total stress is calculated and matrix plastic state is updated. Constitutive tensors of fiber, matrix, and composites are calculated, and the determinant in (5) is evaluated. This procedure is similar to the widely used finite element buckling analysis which is often applied to the analysis of buckling of the structures. When the determinant in (5) becomes approximately equal to zero, the failure is assumed to initiate in the material. At this increment, the calculation is finished, and the applied compressive stress at this time is recorded as the material strength. The analysis is repeated with changing the amount of applied shear stress, and the results for the relationship between material strength and applied shear 


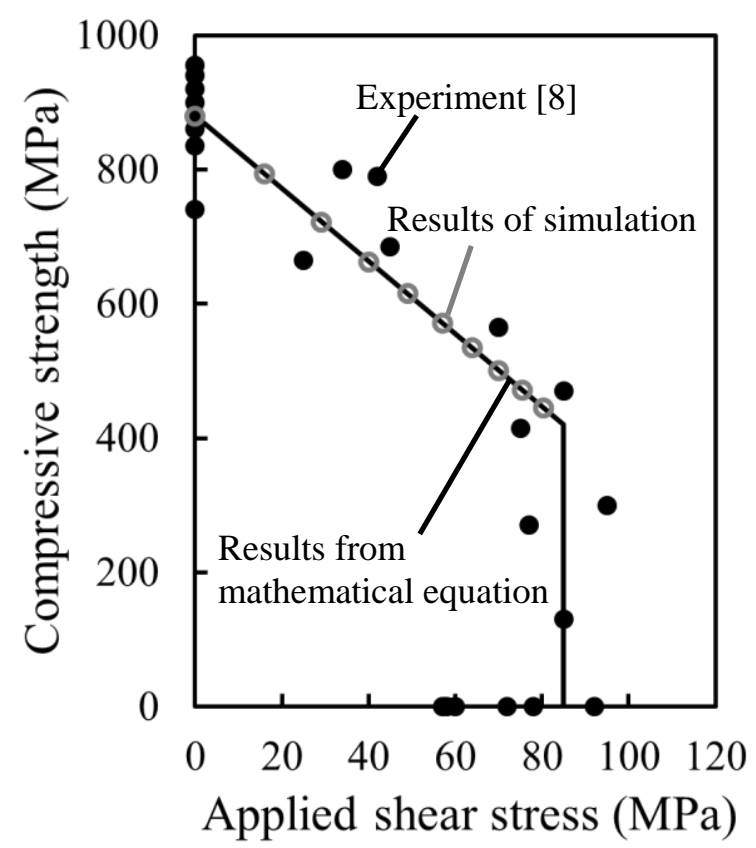

(a) Carbon fiber/epoxy resin T300/BSL914C

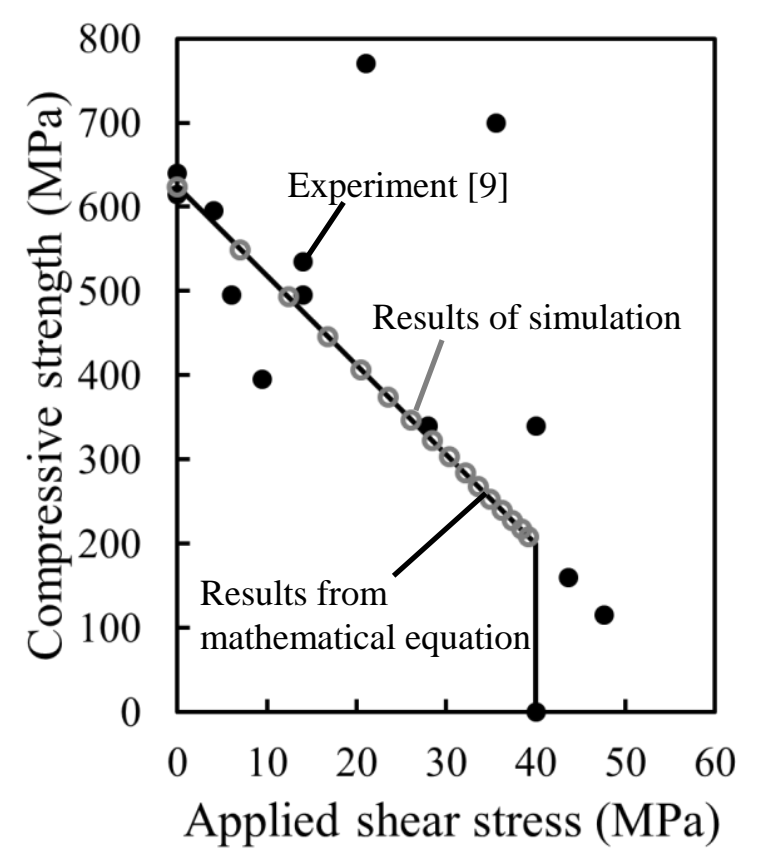

(b) Carbon fiber/vinylester resin IM-7-12K/Derakane 411-C50

Figure 5. Analysis results for relationship between applied shear stress and compressive strength of the material.

stress is obtained. Similar to the previous section, two kinds of composite material carbon fiber/epoxy resin T300/BSL914C and carbon fiber/vinylester resin IM-712K/Derakane 411-C50 are analyzed to compare the analysis results with the experimental results. Fig. 5(a) and (b) show the analysis results for the relationship between the applied shear stress and compressive strength. The analysis results for the relationship between the applied shear stress and compressive strength agree with the results from the simulation and experimental results. As the results of the analysis, when the applied shear stress increases, yielding of matrix occurs at early stage, and then nonlinearity appears in the stress-strain relation of composite material, and the compressive strength of the material reduces.

\section{CONCLUSIONS}

This study investigates numerical analysis for effect of applied shear stress on longitudinal compressive strength of fiber reinforced composite materials. The following remarks are obtained.

1. Results of numerical simulation show that when compressive failure initiates in the material, matrix causes large shear deformation due to nonlinear stressstrain relation of matrix, and the initiation of compressive failure of the material is closely related with the nonlinear stress-strain relation of matrix.

2. The numerical results show that when the applied shear stress increases, the compressive strength of composite materials decreases, and this relation is close to the linear relation.

3. From the results of the analysis using mathematical equations, when the applied shear stress increases, yielding of matrix occurs at early stage, and then nonlinearity appears in the stress-strain relation of composite material, and the compressive strength of the material reduces.

\section{REFERENCES}

[1] M. J. Hinton and P. D. Soden, "Predicting Failure in Composite Laminates: The Background to The Exercise," Compos. Sci. Technol., vol. 58, pp. 1001-1010, 1998.

[2] C. R. Schultheisz and A. M. Waas, "Compressive Failure of Composites, Part I: Testing and Micromechanical Theories," Prog. Aerosp. Sci., vol. 32, pp. 1-42, 1996.

[3] A. M. Waas and C. R. Schultheisz, "Compressive Failure of Composites, Part II:, Experimental Studies” Prog. Aerosp. Sci., vol. 32, pp. 43-78, 1996.

[4] S. T. Pinho, C. G. Davila, P. P. Camanho, L. Iannucci and P. Robinson, "Failure Models and Criteria for FRP under In-plane or Three-dimensional Stress States Including Shear Non-linearity," NASA Technical Memorandum-2005-213530, 2005.

[5] S. Basu, A. M. Waas, and D. R. Ambur, "Compressive Failure of Fiber Composites Under Multi-axial Loading," J. Mech. Phys. Solids, vol. 54, pp. 611-634, 2006.

[6] J. LLorca, C. González, J. M. Molina-Aldareguía, J. Segurado, R. Seltzer, F. Sket, M. Rodríguez, S. Sádaba, R. Muñoz, and L. P. Canal, "Multiscale Modeling of Composite Materials: a Roadmap Towards Virtual Testing," Adv. Mater., vol. 23, pp. 5130-5147, 2011.

[7] P. D. Soden, M. J. Hinton, and A. S. Kaddour, "Lamina Properties, Lay-up Configurations and Loading Conditions for a Range of Fibre-Reinforced Composite Laminates," Compos. Sci. Technol., vol. 58, pp. 1011-1022, 1998.

[8] M. J. Hinton, A. S. Kaddour, and P. D. Soden, “A Comparison of the Predictive Capabilities of Current Failure Theories for Composite Laminates, Judged Against Experimental Evidence," Compos. Sci. Technol., vol. 62, pp. 1725-1797, 2002.

[9] C. S. Yerramalli, and A. M. Waas, "A Failure Criterion for Fiber Reinforced Polymer Composites Under Combined CompressionTorsion Loading," Int. J. Solids Struct., vol. 40, pp. 1139-1164, 2003.

[10] T. Nadabe, and N. Takeda, "Numerical Simulation and Theoretical Modeling of Longitudinal Compressive Failure in Fiber Reinforced Composite Materials," Proc. International Conference on Logistics Engineering, Management and Computer Science (LEMCS 2014), Atlantis-Press, May 2014, doi:10.2991/lemcs-14.2014.206. 